\title{
Revisão de literatura sobre alimentação e saúde
}

\author{
Priscilla Marcondelli* \\ Antônio Felipe Marangon* \\ Bethsáida de Abreu Soares Schmitz*
}

RESUMO - O objetivo do presente trabalho é, a partir da revisão dos diversos estudos realizados, ressaltar a importância dos hábitos alimentares para a saúde e descrever os métodos existentes de avaliação do consumo alimentar. A rápida mecanização da vida diária traz alguns perigos como as mudanças do comportamento alimentar, que fazem parte do contexto maior da transição nutricional, com alterações quantitativas e qualitativas na dieta. Como exemplo, observa-se o aumento da ingestão energética com um papel maior de gorduras e açúcares adicionados nos alimentos, grande ingestão de gorduras saturadas, principalmente de origem animal, ingestão reduzida de carboidratos complexos e fibras dietéticas e redução da ingestão de frutas e vegetais. Fica claro portanto, que a dieta com toda sua variedade cultural, pode dar indicativos das condições de saúde, crescimento e desenvolvimento dos indivíduos, sendo de fundamental importância a correta caracterização dos hábitos alimentares para uma adequada intervenção e educação nutricional.

Palavras-chave: hábitos alimentares; promoção da saúde; métodos de avaliação do consumo; inquéritos dietéticos.

\section{Eating habits for the health: a review}

\begin{abstract}
The aim of this paper is to stress the importance of eating habits for the health and describe the existing methods to evaluate the food consumption, based on a revision of several studies. The fast mechanisation of the daily life brings some dangers such as changes in the eating behaviour, which are part of the greater context of the nutritional transition, with qualitative and quantitative alterations with a greater addition of fats and sugar to foods, a greater ingestion of saturated fats (mainly of animal origin), lower ingestion of complex carbohydrates and dietary fibbers and reduction of the ingestion of fruits and vegetables. Therefore, it is clear that a diet with a wide cultural variety may indicate that health conditions, growth and development of individuals, and the correct characterisation of eating habits is of crucial importance for an adequate intervention and nutritional education.
\end{abstract}

Key words: eating habits; health promotion; methods to evaluate food consumption; inquiries on diets.

*Professores da Faculdade de Ciências da Saúde do UniCEUB. 
O organismo adquire a energia e os nutrientes necessários para um bom estado de saúde, mediante uma dieta adequada em quantidade e qualidade de alimentos. Logo, já são conhecidos há muito tempo, os prejuízos decorrentes do consumo alimentar insuficiente ou excessivo (Monteiro, 1995).

A rápida mecanização da vida diária traz perigos. Estes incluem mudança do comportamento, como sedentarismo, dietas desequilibradas em calorias, colesterol e sal, fumo, além de um ambiente deteriorante, em função da poluição do ar, exposição a agentes químicos e biológicos, contaminação do solo e água e riscos de intoxicação alimentar. Estes fatores resultam em um aumento das doenças pulmonares, coronárias e câncer (WHO, 1997).

O predomínio da dieta denominada ocidental, rica em gorduras, açúcares e alimentos refinados, e reduzida em carboidratos e fibras, vem sendo observado em vários países e regiões do mundo. Paralelo a este quadro, o declínio progressivo da prática de atividade física, contribui para o aumento da incidência de obesidade (Monteiro, 1995). No Brasil, por exemplo, apesar da desnutrição sempre ter sido um fator de risco primário a saúde, nas últimas décadas tem-se observado uma transição do déficit para o excesso de alimentação (Doyle \& Feldman, 1997).

O objetivo do presente trabalho é ressaltar a importância da alimentação para a saúde e caracterizar os métodos de avaliação de consumo alimentar por meio de uma revisão bibliográfica abrangendo esses temas.

\section{A influência dos hábitos alimentares sobre a saúde}

O estudo dos hábitos alimentares através do tempo é complexo. Esta complexidade se relaciona à disponibilidade de milhares de alimentos em qualquer ponto no tempo, e a uma ampla variedade de formas e modos como estes são preparados e consumidos (Popkin e cols., 1999).

Além disso, o estudo das tendências no consumo de alimentos deve também considerar mudanças no suprimento de alimentos, expandindo os locais onde são servidos e consumidos, tanto quanto onde acontece a sua aquisição. Deve-se considerar que o comportamento do consumidor, as suas atitudes e seus conhecimentos relacionados com a dieta e a saúde também mudam através do tempo.

Com o aumento das evidências científicas que dão suporte à visão de que alterações na dieta influenciam, tanto positiva, quanto negativamente a saúde, a nutrição está se tornando o maior determinante, que pode ser modificado para a prevenção de doenças crônicas não transmissíveis. Alterações dietéticas podem não somente influenciar a saúde presente, mas também determinar ou não a ocorrência futura de patologias como câncer, doenças cardiovasculares e diabetes (WHO, 2003). 
O aumento da urbanização do mundo está distanciando mais as pessoas da produção primária dos alimentos e tem conseqüências negativas nos hábitos alimentares e estilo de vida dos indivíduos. Mudanças na dieta, tipo de trabalho e lazer - freqüentemente referidas como transição nutricional - estão contribuindo para os fatores causais de doenças. Essas mudanças parecem estar se acelerando, especialmente nos países em desenvolvimento (WHO, 2003).

As mudanças dietéticas que caracterizam a transição nutricional incluem alterações quantitativas e qualitativas na dieta, como por exemplo, o aumento da ingestão energética com um papel maior de gorduras e açúcares adicionados nos alimentos, grande ingestão de gorduras saturadas, principalmente de origem animal, ingestão reduzida de carboidratos complexos e fibras dietéticas e redução da ingestão de frutas e vegetais (Drewnowski \& Popkin, 1997).

Em 2001, as doenças crônicas não transmissíveis contribuíram aproximadamente com $60 \%$ do total de mortes reportados no mundo e quase metade disso foi atribuída a doenças cardiovasculares. Obesidade e diabetes também estão mostrando tendências preocupantes não somente por já afetarem uma grande parte da população, como também devido ao aparecimento precoce na vida das pessoas (WHO, 2002).

\section{Obesidade}

A obesidade é a forma mais comum de má-nutrição, contribuindo para o surgimento de diversas doenças decorrentes do excesso de peso corporal, dos hábitos alimentares inadequados e da resistência periférica à insulínica (Flegal e cols., 2002; NIH, 1998). Nos Estados Unidos, a prevalência de obesidade em mulheres adultas é de 33,4\% e, em homens, 27,5\% (Flegal e cols., 2002)

Estudos demonstram que a obesidade já pode ser considerada uma pandemia, pois afeta todos os países do mundo (WHO, 1997). O número de casos de obesidade tem aumentado significativamente nos últimos anos, considerando-se hoje um caso de saúde pública (WHO, 1997; Raman, 2002). Observa-se este aumento não somente nos países desenvolvidos, como Estados Unidos, mas também nos países em desenvolvimento como o Brasil, onde também há o aumento significativo de obesos. Segundo a Sociedade Brasileira de Cardiologia, 32\% dos adultos brasileiros são obesos (Sociedade Brasileira de Cardiologia 1999 a,b).

A obesidade aumenta o risco de várias alterações no organismo humano, sendo as principais a hipertensão arterial, o Diabetes Mellitus, as doenças cardiovasculares, a resistência à insulina, o cálculo na vesícula e colecistes, a apnéia do sono e outras disfunções respiratórias, além do aumento da incidência de alguns 
tipos de câncer (Pi-Sunyer, 2002; WHO, 2000).

O sobrepeso em adultos também está associado ao aumento do risco de desenvolvimento de algumas doenças crônicas não transmissíveis, como demonstrado num estudo longitudinal com duração de 10 anos, no qual a incidência de Diabetes Mellitus, hipertensão arterial, doenças coronarianas, câncer de cólon e próstata aumentaram de acordo com o grau de sobrepeso tanto em homens como em mulheres (FIELD e cols., 2001). Além destas consequiências, a obesidade está relacionada com problemas sociais e econômicos, como gastos excessivos com medicamentos e serviços de saúde e exclusão social (Nicklas, 2001; Raman, 2002).

O nível de escolaridade também está diretamente associado à obesidade, pois indivíduos que possuem menor nível escolar apresentam maiores riscos de desenvolverem sobrepeso e obesidade, conseqüentemente os riscos das doenças associadas à obesidade também são maiores entre estes (Mokdad e cols., 2003).

As alterações dos hábitos alimentares têm sido marcadas pelo aumento do conteúdo de lipídios na dieta, o que contribui significativamente no aumento da incidência da obesidade (Flatt \& Tremblay, 1998).

Corroborando com esse estudo, Westerterp-Plantega e cols. (1998) observaram que indivíduos obesos, quando comparados aos não obesos, apresentavam uma tendência a consumir maior quantidade de alimentos de alta densidade energética, principalmente com alto conteúdo de lipídios, e relativamente menor quantidade de alimentos de baixa densidade energética (frutas e vegetais).

Morris e Zemel (1999) mostraram que o maior conteúdo de carboidratos na dieta, principalmente na forma simples, representa um fator de risco para o desenvolvimento da obesidade.

\section{Diabetes}

Nos EUA, a prevalência de diabetes tipo II ficou seis vezes maior considerando o período de 1958 à 1993, e em 1996 matou 193.000 americanos (American Diabetes Association, 1996). Este tipo de diabetes não pode mais ser considerado uma patologia de adultos, pois sua incidência aumentou 10 vezes em adolescentes (19821994), com 33\% de casos diagnosticados em 1994 em adolescentes de 10-19 anos, comprovando que o aumento do diabetes não reflete somente o fato de que a população americana está vivendo mais (Pinhas, Hamiel e cols., 1996).

O diabetes tipo I também pode ser chamada de diabetes insulino dependente, ao passo que a tipo II não possui a necessidade de insulina exógena, sendo chamada, dessa forma, de insulino independente. O diabetes tipo II acomete aproximadamente $90-95 \%$ dos casos diagnosticados com obesidade e, inicia-se com resistência à 
insulina, associada à idade avançada, sobrepeso, histórico familiar, intolerância à glicose e inatividade física. Entretanto, o diabetes tipo II tem aumentado principalmente em crianças e adolescentes (American Diabetes Association, 2000). Quanto maior for o número de fatores de risco apresentados pelo indivíduo, maior será a chance de desenvolver Diabetes Mellitus (American Diabetes Association, 2003).

Em 2000, 17 milhões de pessoas apresentavam diabetes nos EUA, o que correspondia à 6,2 \% da população, sendo que 11,1 milhões delas já tinham sido diagnosticadas, mas 5,9 milhões ainda não apresentavam diagnóstico (American Diabetes Association, 2000). Espera-se que a prevalência de Diabetes Mellitus aumente para 9\% em 2025 (American Diabetes Association, 2003).

Outros pontos importantes na prevenção do diabetes merecem atenção em pesquisas futuras, como os fatores envolvidos na obesidade, dietas, atividade física, adoção e manutenção de dietas saudáveis, controle do peso, além de vários fatores comportamentais (Wing e cols., 2001).

Observa-se que a perda de peso ( $5-10 \%$ do peso corporal) e a prática de atividade física (30 minutos diários) já possuem excelentes benefícios. Mudança no estilo de vida deveria ser o primeiro passo na prevenção dessa doença, segundo a Associação Americana de Diabetes (American Diabetes Association, 2003).

No tratamento do diabetes a dieta exerce um papel primordial para o seu controle. Tem como objetivos fornecer calorias suficientes para a manutenção do peso saudável em plena atividade; manter equilíbrio entre a ingestão de alimentos e a insulina disponível; fornecer alimentação nutricionalmente equilibrada, de acordo com as necessidades individuais; manter bom controle metabólico para prevenir e/ou retardar complicações agudas e crônicas; e proporcionar uma melhor qualidade de vida (Batista, e cols.., 1996; Brasil, 2000).

O consumo de fibras solúveis é aconselhado devido às propriedades que tornam a absorção de glicose mais lenta, retardam a hidrólise do amido, melhoram a tolerância à glicose, controlando o diabetes tipo II, além de diminuírem a ingestão calórica (McGrowan, 1997).

O consumo de bebidas alcoólicas pode interferir negativamente no controle do diabetes, alterando o metabolismo glicídico, além de possivelmente elevar as concentrações dos triglicerídeos séricos (American Heart Association, 2001 b).

Quanto ao consumo de açúcar, para uma dieta saudável que possa contribuir para a prevenção das principais doenças crônicas não transmissíveis, recomendase que os valores para o consumo de açúcar não exceda $10 \%$ do total das calorias diárias (WHO, 2003). 


\section{Doenças cardiovasculares}

As dislipidemias são alterações nos níveis sanguíneos dos lipídeos circulantes, podendo ser de dois tipos básicos: hipercolesterolemia e hipertrigliceridemia (Cuppari, 2002).

O Consenso Brasileiro sobre Dislipidemias (Sociedade Brasileira de Cardiologia, 1996) estabelece quatro situações onde os lipídeos sanguíneos estão elevados: na hipercolesterolemia há a elevação do colesterol total; na hipertrigliceridemia os triglicerídeos estão aumentados; na hiperlipidemia mista há a elevação do colesterol total e dos triglicerídeos; e por fim, pode ocorrer a redução isolada da lipoproteína de alta densidade (HDL-c) em associação com o aumento da lipoproteína de baixa densidade (LDL-c).

O aumento da concentração sanguínea dos lipídeos pode causar doenças nas artérias coronárias (DAC), xantomas, arco-coreano, pancreatite, além de manifestações metabólicas como hiperuricemia e intolerância à glicose (Sociedade Brasileira de Cardiologia, 1996).

A alimentação, portanto influi diretamente nas doenças cardiovasculares. Estudos epidemiológicos mostram a relação entre o consumo de grande quantidade de gordura saturada, colesterol, sal e carboidratos refinados e o alto risco para doenças coronarianas (WHO, 2003), sendo que a diminuição dessa ingestão também reduz a incidência de doenças coronarianas em 24\% (Gordon, 1995a; Gordon, 1995b).

Os ácidos graxos saturados (AGS) são a principal causa alimentar de elevação de colesterol plasmático e o consumo do colesterol e dos AGS aumenta o LDL-C. Para reduzir a ingestão de colesterol e AGS, deve-se restringir principalmente o consumo de alimentos de origem animal (carnes gordurosas, leite integral e derivados) (American Heart Association, 2000).

Os ácidos graxos insaturados (AGI) são representados pelas séries ômega6 (linoléico e araquidônico), ômega-9 (oléico) e ômega-3 (a-linolênico, icosapentaenóico-EPA e docosahexaenóico-DHA). Os ômega-6 são encontrados nos óleos vegetais, exceto os de coco, cacau e dendê. A substituição isocalórica dos AGS por AGI reduz o LDL-C e o HDL-C. Os AG monoinsaturados (ácido oléico) reduzem o LDL-C, sem, no entanto, diminuir o HDL-C. Suas principais fontes são os óleos de oliva e canola, azeitonas, abacate e oleaginosas (castanhas, nozes, amêndoas) (American Heart Association, 2000).

Os ômega-3 têm como efeito farmacológico a diminuição da trigliceridemia e do colesterol plasmático, a melhora da função plaquetária e a promoção de uma redução na pressão arterial. Como fontes de ômega- 3 encontram-se peixes de água fria como a cavala, a sardinha, o salmão, o atum e o arenque (EPA e DHA) e óleos 
de soja e canola (ácido linolênico) (American Heart Association, 2000).

Os ácidos graxos trans (trans-isômeros) são sintetizados durante o processo de hidrogenação dos óleos vegetais na produção de margarinas e elevam o LDLC e reduzem o HDL-C (Lichtenstein e cols. 1999). As principais fontes são margarinas duras, óleos e gorduras hidrogenadas, biscoitos, bolos, pães, pastéis, batatas chips e sorvetes cremosos (Lichtenstein e cols. 1997).

As fibras solúveis encontradas nos legumes, aveia, leguminosas e algumas frutas estão relacionadas aos benefícios diretos aos sujeitos com doenças cardiovasculares, pois atuam na redução das concentrações séricas do LDL e na eliminação do colesterol. Uma teoria que explicaria esse processo é de que essas fibras aumentariam a excreção de ácidos biliares fazendo com que o fígado removese o colesterol sanguíneo para a síntese de novos ácidos e sais biliares. Outra hipótese indica que um produto da fermentação das fibras solúveis, o propionato, inibiria a síntese hepática do colesterol. Embora esta teoria ainda não tenha se confirmado, o papel preventivo de diferentes fibras na redução do colesterol plasmático vem se confirmando cada vez mais (Gallaher \& Schneeman, 1997).

A recomendação da American Heart Association de ingestão de fibra alimentar total para adultos é de 20 a $30 \mathrm{~g} /$ dia, sendo em torno de $25 \%$ (6g) de fibra solúvel (American Heart Association, 2000).

No quadro a seguir podem ser observadas as metas dietéticas indicadas pela American Heart Association (2001a) e no Brasil pela Sociedade Brasileira de Cardiologia (2001) para o tratamento e a prevenção dos distúrbios lipídicos, onde é claro a diferenciação entre os tipos de gordura, incluindo a preocupação com ácidos graxos insaturados, ao invés de somente observar o total de gordura. Uma atenção especial deve ser dada aos carboidratos que deveriam vir principalmente de alimentos ricos em carboidratos complexos como grãos integrais, frutas e vegetais, confirmando a importância das fibras alimentares.

Tabela 1: Recomendações dietéticas para o tratamento das hipercolesterolemias.

\begin{tabular}{cc}
\hline Nutriente & Consumo Recomendado \\
\hline Gordura total & $25-35 \%$ das calorias totais \\
Ácidos Graxos Saturados & $<7 \%$ das calorias totais \\
Ácidos Graxos Polinsaturados & $>10 \%$ das calorias totais \\
Ácidos Graxos Monoinsaturados & $>20 \%$ das calorias totais \\
Carboidratos & $50-60 \%$ das calorias totais \\
Proteínas & Aproximadamente $15 \%$ das calorias totais \\
Colesterol & 200 a 30 g/dia \\
Fibras & Calorias para atingir e manter o peso desejável \\
\hline
\end{tabular}

Fontes: American Heart Association, 2001a; Sociedade Brasileira de Cardiologia, 2001. 
É importante também ressaltar que o consumo moderado de álcool tem um efeito protetor nas doenças cardiovasculares, por meio do aumento do HDL e da redução do fibrinogênio (Kotchen \& Kotchen, 1998). A American Heart Association (2001b) sugere limitar o consumo de bebidas alcoólicas para 1 dose para as mulheres e duas doses para os homens. Uma dose corresponde a 14 gramas de álcool e pode ser definida como uma lata de cerveja ou 1 copo de vinho (120ml). O consumo de doses maiores tem sido relacionado a efeitos adversos relacionados às cardiopatias como hipertensão, arritmia, derrame hemorrágico e morte súbita.

\section{Hipertensão}

A hipertensão arterial, com causa multifatorial, é conceituada como uma síndrome caracterizada pela presença de níveis tensionais elevados, associados a alterações metabólicas e hormonais e a fenômenos tróficos (hipertrofia cardíaca e vascular) (Sociedade Brasileira de Hipertensão Arterial, 1998).

Este é um dos mais importantes fatores de risco para o desenvolvimento das doenças cardiovasculares, explicando $40 \%$ das mortes por acidente vascular encefálico e $25 \%$ daquelas por doença arterial coronariana (Burt e cols., 1995).

A pressão sanguínea elevada aumenta os riscos para derrame cerebral, doenças cardiovasculares e renais, além de falência cardíaca (Miller, 2003). Mais de 50 milhões de americanos têm pressão sanguínea acima de 140/90 mmHg (Burt e cols., 1995). Além do uso de medicamentos, a pressão sanguínea pode ser reduzida por meio do aumento da atividade física, redução do peso corporal, consumo de dieta adequada, redução do consumo de álcool e de sódio (Vollmer e cols., 2001).

Além desse efeito deletério à saúde, a ingestão de altos níveis de sódio na alimentação também aumenta a excreção de cálcio pela urina, causando uma alteração no balanço desse mineral (NIH, 1994). As taxas de prevalência na população urbana adulta brasileira em estudos selecionados variam de 22,3\% a 43,9\% (Freitas e cols., 2001; Fuchs e cols., 1995; Martins e cols., 1997; Rego e cols., 1990).

O excesso de peso aumenta de duas a seis vezes o risco de hipertensão, enquanto a diminuição de peso em normotensos reduz a pressão e a incidência de hipertensão. Como medida preventiva, recomenda-se a manutenção do peso ideal (The trials of Hypertension Prevention Collaborative Research Group, 1992).

A dieta habitual do brasileiro contém 10 a $12 \mathrm{~g}$ de sal/dia. É saudável ingerir até $6 \mathrm{~g} /$ dia de sal (4 colheres das de café rasas de sal (4 g) e $2 \mathrm{~g}$ de sal presente nos alimentos naturais), reduzindo o sal adicionado aos alimentos, evitando o saleiro à mesa e alimentos industrializados (Sacks, 2001).

A Organização das Nações Unidas para a Agricultura e Alimentação (FAO) 
e a Organização Mundial de Saúde (OMS), em seu relatório "Diet, Nutrition and Prevention of Chronic Diseases" (WHO, 2003) apontam o sal e o consumo de sódio como fatores diretamente relacionados com os níveis de pressão sangüínea e a prevalência de hipertensão em muitas populações. Além do excesso do sódio, a carência de minerais como o potássio, o cálcio e, possivelmente, o magnésio, vem sendo associada a níveis mais altos de pressão arterial, o que reforça a necessidade de dietas que contenham quantidades adequadas de frutas, vegetais e laticínios com baixo teor de gordura. Os substitutos do sal contendo cloreto de potássio e menos cloreto de sódio (30\% a 50\%) são úteis para reduzir a ingestão de sódio e aumentar a de potássio (American Heart Association, 1998).

Essa doença não é privilégio de adultos. As crianças também estão sendo acometidas pela hipertensão arterial e isso se deve a má alimentação que predomina nas famílias (Brouhard, 1995). Almeida e cols. (2002) constataram que a exposição de 30 segundos a comerciais de alimentos pode influenciar a escolha de crianças e que no Brasil adolescentes passam cerca de 5 horas por dia diante da televisão. Observou-se que 22,47\% dos comerciais veiculados pela televisão são de alimentos, sendo que desses produtos, a maioria possui um elevado nível de gordura, óleo, açúcar e sal, como os salgadinhos fritos e batata chips. Cada hora de exposição representa um aumento de $2 \%$ na prevalência de obesidade e, conseqüentemente, um maior risco de hipertensão.

Desta forma, a importância da determinação rotineira dos níveis pressóricos na infância tem ganhado importância nas últimas décadas, na medida em que esta conduta possibilitará diagnosticar precocemente as alterações da pressão arterial e conduzir de maneira favorável um maior número de casos (Toporovsky, 1982).

\section{Câncer}

O câncer é atualmente a maior causa de mortalidade no mundo, superado somente pelas doenças cardiovasculares. Em 2000 foram estimados 10 milhões de novos casos e cerca de 6 milhões de mortes no mundo (Parkin, 2001). Essa doença é causada por vários fatores, sendo que a dieta, a inatividade física, o álcool, fatores hormonais e radiações são fatores de risco para o desenvolvimento do câncer (WHO, 2003).

Os fatores dietéticos são responsáveis por cerca de 30\% dos tipos de câncer em países industrializados, fazendo da dieta a segunda causa de câncer que teoricamente pode ser prevenida, após o tabaco, que é a primeira causa (Doll \& Peto, 1996). Por outro lado, estima-se que 32\% dos tipos de cânceres podem ser evitados com mudanças na dieta (Willett, 1995). 
Embora muitas linhas de pesquisas e suas evidências sugerem que a restrição calórica pode contribuir para baixas taxas de câncer de mama, isto não traduz diretamente a principal prática de prevenção. Além disso, recentes estudos indicam que a carne vermelha ocupa um papel importante no desenvolvimento de câncer de cólon e próstata, embora, não inteiramente devido ao teor de gordura (Willett, 1995).

Evidências indicam que no câncer de mama, o conceito de que a ingestão de gordura, por si só, é a razão primária para a diferença nas taxas entre os países, não tem sido apoiada por estudos prospectivos. Levando em consideração que os efeitos adversos da gordura e de outros componentes relacionados ao câncer estão em estudo, a maior evidência da última década tem indicado a importância do efeito protetor das frutas e dos vegetais (Willett, 1995).

\section{Cálculo renal}

A incidência de calculo renal é de $12 \%$ nos EUA e a taxa de recaída é ainda muito alta (Fine e cols., 1995). A fim de evitar ocorrência de cálculo, as recomendações dietéticas abaixo têm mostrado um efeito protetor, como a alta ingestão de líquidos (mais de 2 litros por dia) (Borghi e cols., 1999); a moderada ingestão de sódio (2$3 \mathrm{~g}$ dia); a limitada ingestão de proteína animal; e a limitação da vitamina C no máximo em $2 \mathrm{~g}$ dia. A restrição de cálcio rigorosa deveria ser evitada (Pearle, 2001). No caso dos líquidos, contribuem para a prevenção de formação de cálculos por reduzir a saturação de oxalato de cálcio e aumentar a resistência da urina da sobrecarga de oxalato (Borghi e cols., 1999). No caso das proteínas, o excesso de proteína animal pode aumentar o risco de cálculos pelo aumento do ácido úrico urinário devido à sobrecarga de purina e pela redução da hipercalciúria proveniente da carga ácida (Martini e Wood, 2000).

No caso do cálcio, estudos têm mostrado que dietas ricas em cálcio não alteram as chances de cristalização de oxalato de cálcio em indivíduos sadios, mesmo com o aumento urinário do cálcio causado por outros ajustes na dieta relativamente à ingestão de líquidos, potássio e fosfato (Heller e cols., 2003).

Curham (2002) observou que a reincidência de cálculos renais foi reduzida no período de 5 anos com uma ingestão normal de cálcio, baixa ingestão de proteína e sal. Borghi e cols. (2002) também observaram que a ingestão normal de cálcio, a restrição de proteína animal e sal, proporcionavam maior proteção contra a recorrência de pedra nos rins em homens com recorrentes pedras de oxalato de cálcio e hipercalciúria. Reddy e cols. (2002) também realizaram um estudo a curto prazo mostrando que dietas ricas em proteínas e baixas em carboidratos, usadas para a redução de peso, promovem carga excessiva de ácido, possivelmente aumentando o risco de formação de cálculos nos rins e perda óssea. 


\section{Métodos de avaliação de consumo alimentar}

A avaliação do consumo alimentar é provavelmente o maior indicador indireto usado para se verificar o estado nutricional. Diferentes métodos são descritos na literatura: o recordatório 24 horas, o diário alimentar, o questionário de frequiência de alimentos e a história alimentar, entre outros (Gibson, 1990). Limitações existem entre os diversos métodos de obtenção de informações sobre consumo de alimentos em participantes vivendo livremente (New Developments in Dietary Assessment, 1997), e existe atualmente uma dúvida se realmente alguns desses métodos poderiam ser considerados padrão-ouro em termos de validade, pois cada um tem suas vantagens e desvantagens (Mertz, 1992).

O recordatório 24 horas foi selecionado como o primeiro instrumento para avaliação do consumo alimentar no $3^{\circ}$ National Health and Nutrition Examination Survey (NHANES III) (Sempos e cols., 1992). É um método de baixo custo e de rápida administração (20 minutos ou menos) e pode fornecer informações detalhadas de alimentos específicos (Block, 1989; Feskanich \& Willet, 1993). Esse método é considerado mais objetivo que a história dietética e o questionário de freqüência alimentar e sua administração não altera a dieta habitual. No entanto, recordatórios têm algumas limitações como, por exemplo, falhas de memória e fornecimento de dados de um consumo alimentar não usual, já que o indivíduo pode ter consumido alimentos atípicos no dia anterior à entrevista. Múltiplos recordatórios 24 horas realizados em tempos espaçados podem fornecer uma razoável estimativa da ingestão de nutrientes de um indivíduo (USA, 1989; Block, 1989; Block, 1992)

Outro método muito utilizado para coleta de dados alimentares individuais é o diário alimentar, que não depende da memória porque os alimentos e bebidas são registrados no mesmo período da ingestão. Consiste em um registro escrito de todas as comidas e bebidas consumidas pelo respondente, realizado em 3, 4 ou 7 dias, podendo ser levado em consideração o final de semana. Além disso, pode fornecer dados detalhados do consumo alimentar com informações importantes sobre hábitos alimentares. Vários diários devem ser realizados em diferentes períodos para alcançar estimativas da ingestão alimentar usual (Tarasuk \& Beaton, 1992; Larkin e cols., 1991). Esse método requer uma grande cooperação do respondente que deve ser capaz de gastar tempo e esforço necessários para o correto preenchimento do diário. $\mathrm{O}$ ato de registrar a ingestão alimentar durante vários dias pode influenciar na dieta do respondente, causando algumas alterações como simplificar seus alimentos e bebidas para facilitar o processo de registro (Feskanich \& Willet, 1993; Dwyer,1994)

O questionário de freqüência alimentar é um método de consumo alimentar que consiste de uma lista de aproximadamente 100 ou menos alimentos individuais 
ou grupos de alimentos que são importantes na ingestão de energia e nutrientes da população. Os respondentes indicam quantas vezes por dia, semana, mês ou ano eles consomem aqueles alimentos (Block, 1992; Feskanich \& Willet, 1993).

O questionário de frequiência de alimentos, por exemplo, tende a superestimar o consumo de alimentos (Serra-Majem e cols., 1994), porém mostra-se um instrumento proveitoso por poder ser auto - administrado, processado rapidamente, e ser de baixo custo (Willett, 1998).

Para o uso em uma grande intervenção como para grupos de um ambiente comunitário, vários questionários de freqüência alimentar têm sido desenvolvidos para perguntar o que a pessoa consumiu no dia, mês e ano num determinado período (Field e cols., 1998; Willett, 1998, Hunt e cols., 1998).

A validade e reprodutibilidade deles estão sendo investigadas em detalhes e comparadas ao padrão ouro tais como múltiplos recordatórios de 24 horas coletados em dias não consecutivos ou registros alimentares de 4 a 7 dias (Baranowiski e cols., 1997; Willett, 1998). Define-se reprodutibilidade como "a habilidade de um método produzir a mesma estimativa em duas ou mais ocasiões, assumindo que nada mudou no seu interim" (autor, ano e página) e validade como "a habilidade de um instrumento medir de fato o que se pretende medir" (autor, ano e página) (Lee e Nieman, 1996). Para recordatórios alimentares e registros, existe uma discussão de quantos e quais dias são necessários para alcançar suficiente validade e reprodutibilidade (Willett, 1998).

A história alimentar é usada para avaliar a ingestão dietética usual de um indivíduo, em um longo período de tempo, como o mês ou o ano passado, por meio de uma entrevista com um profissional treinado. É um dos métodos preferidos na obtenção de estimativa de ingestão dietética por detectar mudanças sazonais e facilitar a obtenção de todos os nutrientes ingeridos. Existe uma grande limitação que é o tempo necessário para responder a entrevista que vai de 1 a 2 horas, tornandoo um método caro. Além disso, os nutrientes tendem a ser superestimados (Block, 1989; Bittoni \& Wilkins, 1994).

O dia da semana também influencia o consumo alimentar. Beaton e cols.. (1979) mostraram que mulheres consumiam mais alimentos aos domingos do que durante a semana. Portanto, quando se analisa o consumo alimentar apenas de um único dia, não se considera as variações diárias existentes na rotina do ser humano.

Apesar de a pirâmide alimentar brasileira (Philippi e cols., 1999) não ser um método específico de avaliação do consumo e sim um guia alimentar, o uso das suas porções pode ser indicado na tentativa de se avaliar o consumo alimentar dos indivíduos. Ela foi desenvolvida a partir da pirâmide criada pelo departamento 
de agricultura dos EUA (USDA, 1992) e contém orientações sobre a freqüência ideal de consumo de grupos de alimentos e suas porções, facilitando a obtenção de informações qualitativas da alimentação, refletindo o valor energético total da dieta; além de conter os três conceitos das diretrizes dietéticas: variedade, moderação e proporcionalidade (Achterberg e cols., 1994). Desde a época em que foi desenvolvida, a pirâmide alimentar tem sido largamente utilizada em comunidades saudáveis como uma ferramenta educacional (Cleveland e cols., 1997), empregada principalmente em faculdades e escolas (Tavelli ecols 1998).

Em pesquisa realizada por Pereira (1999) com freqüentadores de academias de ginástica, $42.6 \%$ dos participantes não consumiram nenhum alimento do grupo das frutas e $27.7 \%$ não consumiram nenhum alimento do grupo dos vegetais de acordo com a Pirâmide dos Alimentos (USDA, 1992); dados estes importantes na caracterização e diagnóstico do consumo alimentar do grupo estudado. Portanto, estudos simples que usem os grupos da Pirâmide dos Alimentos podem efetivamente gerar informações relevantes sobre o consumo alimentar da população estudada.

\section{Comentários}

A dieta das pessoas, com toda sua variedade cultural, pode indicar características relativas à saúde e condições de crescimento e desenvolvimento. Comportamentos de risco, como o uso de tabaco e inatividade física, podem modificar este resultado para melhor ou pior. Como prevenção e tratamento das doenças crônicas não transmissíveis, deve-se diminuir o consumo de gorduras saturadas, colesterol, ácidos graxos trans e açúcar e aumentar o consumo de fibras e vitaminas com função anti-oxidante.

Fica claro, portanto, a importância da correta caracterização da dieta na promoção da saúde da comunidade, na posterior intervenção por meio da educação nutricional e na avaliação da eficácia de programas.

Neste sentido, a metodologia e o conhecimento relacionados à determinação do estado nutricional necessitam de maiores avanços. Desse modo, estudos epidemiológicos que relacionam nutrição e saúde têm feito uso de dados de ingestão alimentar no intuito de procurar estabelecer as devidas associações entre estes fatores. 


\section{Referências}

ACHTERBERG, C.; MCDONNELL, E. \& BAGBY, R. How to put the food guide pyramid into practice. J Am Diet Assoc, 94: 45-56. 1994.

ALMEIDA, S. S.. e cols.. Quantidade e qualidade de produtos alimentícios anunciados na televisão brasileira. Rev. Saúde Pública, v. 36, n. 3, p. 353-5, 2002.

AMERICAN DIABETES ASSOCIATION. Diabetes 1996: Vital Statiscs. Alexandria, VA:

American Diabetes Association, 1996.

AMERICAN DIABETES ASSOCIATION. National Diabetes Fact Sheet. [http:// www.diabetes.org/main/info/facts/facts_natl.jsp (Acessado em 3/31/03). 2000.

AMERICAN DIABETES ASSOCIATION. The Prevention or Delay of Type 2 Diabetes. Diabetes Care; 26: S62-S69, 2003.

AMERICAN HEART ASSOCIATION. AHA Scientific Advisory. A statement for health care professionals from The American Heart Association nutrition committee. Am J Clin Nutr; 98: 1249-53, 1998.

AMERICAN HEART ASSOCIATION. AHA Scientific Statement. Dietary Guidelines. Revision 2000. A statement for health care professionals from the nutrition committee of The American Heart Association. Circulation, 102: 2284-99, 2000.

AMERICAN HEART ASSOCIATION. AHA Scientific Statement: Summary of the scientific conference on dietary fatty acids and cardiovascular health. Conference summary from the nutritiion committee of The American Heart Association. Circulation, 103:7:1034-9, 2001a.

AMERICAN HEART ASSOCIATION. AHA Science Advisory. Wine and your heart. A science advisory for healthcare professionals from the nutritiion committee, council on cardiovascular nursing of The American Heart Association. Circulation, 103:3: 472-5. $2001 \mathrm{~b}$.

BARANOWISKI, T.; BARANOWISKI, J.; DOYLE, C., e cols.. Towards reliable estimation of servings of fruit and vegetables and fat practices from adults' 7- day food records. J Nutr Educ.; 29: 321- 326, 1997.

BATISTA, S. M. M.; FIATES, G. M. R; LÚCIO, L.. Estudo dos hábitos alimentares de diabéticos tipo I. Rev. Ciên. Saúde: v.15, n.1/2, 151-62, jan-dez. 1996.

BEATON, G.H.; MILNER, J.; COREY, P. e cols.. Sources of variance in 24-hour dietary recall data: implications for nutrition study design and interpretation. Am J Clin Nutr. Dec; 32(12): 2546-9, 1979

BITTONI, M.A. \& WILKINS, J.R. 3rd.Assessment of the reliability of a diet history questionnaire. Nutr Cancer, 21:143-55, 1994.

BLOCK, G. Human dietary assessment: methods and issues. Preventive Medicine, 18: 65360, 1989.

BLOCK, G. Dietary assessment issues related to cancer for NHANES III. 
Vital Health Stat Mar;(27):24-31. 1992

BORGHI, L.; MESCHI, T.; SCHIANCHIT, L.; BRIGANTI, A.; GUERRA, A.; ALLEGRI, F. \& NOVARINI, A. Urine volume: stone risk factor and preventive measure. Nephron, $81: 31-37,1999$.

BORGHI, L.; SCHIANCHI, T.; MESCHI, T.; GUERRA, A.; ALLEGRI, F.; MAGGIORE, U. \& NOVARINI, A. Comparison of Two Diets for the Prevention of Recurrent Stones in Idiopathic Hypercalciuria. N Engl J Med, 346: 77-84, 2002.

BROUHARD BH. Hypertension in children and adolescents. Cleve Clin J Med.;62(1):21-8, 1995.

BRASIL, Ministério da Saúde. Abordagem Nutricional em Diabetes Mellitus, Brasília, 2000.

BURT, V.L.; CUTLER, J.A.; HIGGINS, M.; HORAN, M.J.; LABARTHE, D.; WHELTON, P.; BROWN, C. \& ROCCELLA, E.J. Trends in the prevalence, awareness, treatment, and control of hypertension in the adults US population. Data from the health examination surveys, 1960 to 1991. Hypertension; 26:60-9, 1995.

CLEVELAND, L.E.; COOK, D.A.; SMITH, S.M.K. \& FRIDAY, J. Method for assessing food intakes in terms of servings based on food guidance. Am J Clin Nutr, 65: 1254S63S, 1997.

CUPPARI, L. 2002. Guia de Nutrição. Nutrição Clínica no Adulto. São Paulo:Manole, 2002.

CURHAN, G. A normal calcium, low protein, low salt diet reduced recurrence of renal stones over 5 years. Evid Based Med 7: 152-152 2002.

DOLL, R. \& PETO, R. Epidemiology of cancer. In : Weatherall DJ, Ledingham JGG,Warrell DA, (Eds.). Oxford textbook of medicine. Oxford, Oxford University Press, 197221.1996.

DOYLE, E.I.\& FELDMAN, R.H.L.. Factors affecting nutrition behavior among middleclass adolescents in urban area of Northern region of Brazil. Rev. Saúde Pública, 31(4): 342-50, 1997.

DREWNOWSKI A, POPKIN B.M. The nutrition transition: new trends in the global diet. Nutr Rev. Feb 55(2):31-43, 1997.

DWYER JT. Dietary Assessment. In Shils ME, Olson JÁ, Shike M, (Eds.). Modern Nutrition in health and disease, 8th ed., Philadelphia: Lea \& Febiger., 1994

FESKANICH, D. \& WILLET, W.C. The use and validity of food frequency questionnaires in epidemiologic research and clinical practice. Medicine, Exercise, Nutrition, and Health 2:143-154, 1993.

FIELD, A.E.; COAKLEY, E.H.; MUST, A.; SPADANO, J.L.; LAIRD, N.; DIETZ, W.H.; RIMM, E. \& COLDITZ, G.A. Impact of overweight on the risk of developing common chronic diseases during a 10-year period. Arch Intern Med, 161:1581-6, 2001.

FINE, J.K.; PAK, C.Y.\& PREMINGER, G.M. Effect of medical management and residual fragments on recurrent stone formation following shock wave lithotripsy. J Urol 153:27-32. 1995; 
FLATT, J.P., TREMBLAY, A. Energy expenditure and substrate oxidation. In: Bray, G.A., Bouchard, C., James, W.P.T. (Eds.). Handbook of obesity. New York:Marcel Dekker, p.513-537, 1998.

FLEGAL K.M., CARROLL M.D., OGDEN C.L., JOHNSON C.L. Prevalence and trends in obesity among USA adults, 1999-2000. JAMA, 288:1723-7, 2002.

FREITAS O.C., RESENDE C.F., MARQUES N.J. e cols.. Prevalence of hypertension in the urban population of Catanduva, in the State of Sao Paulo, Brazil. Arq Bras Cardiol, 77(1): 9-21, 2001.

FUCHS F.D., MOREIRA L.B., MORAES R.S. e cols.. Prevalência de hipertensão arterial sistêmica e fatores associados na região urbana de Porto Alegre: estudo de base populacional. Arq Bras Cardiol, 63: 473-9, 1995.

GALLAHER, D.D. \& SCHNEEMAN. B.O. Fibra alimentar. In Ziergler EE, Filer JLJ, (Eds.).. Conocimientos actuales sobre nutrición. $7^{\text {th }} \mathrm{ed}$. Washington:International Life Sciences Institute Press, 95-105, 1997.

GIBSON, R.S. Principles of Nutritional Assessment. New York:Oxford University Press. 1990.

GORDON, D.J. Cholesterol and mortality: what can meta-analysis tells us. In: Gallo LL, (Ed). Cardiovascular Disease 2: Cellular and Molecular Mechanisms, Prevention, and Treatment. New York: Plenum Press: 333-340, 1995a.

GORDON, D.J. Cholesterol lowering and total mortality. In: Rifkind BM, (Ed.). Lowering Cholesterol in High Risk Individuals and Populations. New York:Marcel Dekker Inc;33-47, 1995b.

HELLER, H.J.; DOERNER, M.F.; BRINKLEY, L.J.; ADAMS-HUET, B. \& PAK, C.Y. Effect of dietary calcium on stone forming propensity. J Urol, 169:470-474, 2003.

HUNT, M.K.; STODDARD, A.M.; PETERSON, K.; SORENSEN, G.; HEBERT, J.R. \& COHEN, N. Coparison of dietary assessment measures in the treatwell 5- a - Day Worksite study. J Am Diet Assoc; 98: 1021-1023, 1998.

KOTCHEN, T.A. \& KOTCHEN, M.J. Nutrition, diet and hipertension. In Shils ME, e cols.., (Eds.). Modern nutrition in health and disease. $9^{\text {th }}$ ed. USA: Williams \& Wilkins, 1217-27, 1998.

LARKIN, F.A.; METZNER, H.L. \& GUIRE, K.E. Comparison of three consecutive-day and three random-day records of dietary intake. J Am Diet Assoc. (12):1538-42, 1991.

LEE R.D. \& NIEMAN D.C. Nutritional Assessment. $2^{\text {nd }}$ ed. Boston/Massachussetts: WCB/ McGraw-Hill, 689 p., 1996.

LICHTENSTEIN A.H., AUSMAN L.M., JALBERT S.M., SCHAEFER E.J.. Trans fatty acids, plasma lipid levels, and risk of developing cardiovascular disease. Circulation, 95: 2588-90, 1997.

LICHTENSTEIN A.H., AUSMAN L.M., JALBERT S.M., e cols.. Effects of different forms of dietary hydrogenated fats on serum cholesterol levels. $N$ Engl J Med; 
340:1933-40, 1999.

MARTINS I.S., MARUCCI M.F., VELASQUEZ-MELENDEZ G. e cols.. Doenças cardiovasculares ateroscleróticas, dislipidemias, hipertensão, obesidade e diabete melito em população da área metropolitana da região Sudeste do Brasil. III Hipertensão. Rev Saúde Publica, 31(5): 466-71, 1997.

MARTINI, L.A. \& WOOD, R.J. Should dietary calcium and protein be restricted in patients with nephhrolithiasis? Nutr Rev., 58: 111-117, 2000.

McGROWAN, M.P.. Am I at riskfor developing heart disease? Heart fitness for life. New York: Oxford, 10-25, 1997.

MILLER, N.H. Aggressive Blood Pressure Management. J Cardiovasc Nurs, 18: 108-115. 2003.

MERTZ, W. Food intake measurements: Is there a "gold standard"? J Am Diet Assoc, 92(12): 1463-65, 1992.

MONTEIRO, C.A. Velhos e Novos Males de Saúde no Brasil - A Evolução do país e de suas doenças. São Paulo, Editora Hucitec /NUPENS/USP (Capítulo 14) 247-255, 2 a ed., 1995.

MOKDAD, A.H.; FORD, E.S.; BOWMAN, B.A.; DIETZ, W.H.; VINICOR, F.; BALES, V.S. \& MARKS, J.S. Prevalence of Obesity, Diabetes, and Obesity-Related Health Risk Factors, 2001. JAMA, 289:76-79, 2003.

MORRIS, K.L., ZEMEL, M.B. Glycemic index, cardiovascular disease and obesity. Nutrition Review New York, v.57, n.9, (1), 273-276, 1999.

NEW DEVELOPMENTS IN DIETARY ASSESSMENT,. Abstracts. Am J Clin Nutr,; 65 S: 1295S-314S. 1997.

NICKLAS, T.A.; BARANOWSKI, T. \& CULLEN, W. Eating Patterns, Dietray Quality and Obesity. J Am Coll Nutr, 20 , 6, 599-608, 2001.

$\mathrm{NIH}$-National Institutes of Health. Consensus Development Panel on Optimal Calcium Intakes. NIH Consensus Conference. JAMA; 272: 1942-1948, 1994.

$\mathrm{NIH}$ - National Institutes of Health. Clinical guidelines on identification, evaluation, and treatment of overweight and obesity in adults. The evidence report. Bethesda: National Hearth, Lung and Blood Institute, 1998.

PARKIN, D.M. Global cancer statistics in the year 2000. Lancet Oncology, 2: 533-543. 2001.

PEARLE, M.S. Prevention of nephrolithiasis. Curr Opin Nephrol Hypertens; 10:203-209. 2001.

PEREIRA, R.F. Conhecimento de nutrição e hábitos alimentares de alunos de academias de ginástica na cidade de São Paulo. [Dissertação de Mestrado - FCF/FEA/FSP da USP], 1999.

PI-SUNYER FX. The medical risks of obesity. Obes Surg 12:6S-11S, 2002.

PINHAS-HAMIEL, O.; DOLAN, L.M; DANIELS, S.R.; STANDIFORD, D., KHOURY, P.R. \& ZEITLER, P. Increased incidence of non-insulin-dependent diabetes mellitus 
among adolescents. J Pediatr 128:608-615, 1996.

PHILIPPI, S. T.; RIBEIRO, L.C.; LATTERZA, A.R. \& CRUZ, A.T.R. Pirâmide alimentar adaptada: guia para escolha de alimentos. R.Nutr.Campinas 12: 65-80, 1999.

POPKIN, B.M.; HAINES, P.S. \& SIEGA-RIZ, A.M. Dietary Patterns and Trends in the United States: The UNC-CH Approach. Appetite; 32:8-14, 1999.

RAMAN, R. P. Obesity and Health Risks. J Am Coll Nutr, 21: 134S-139S, 2002.

REDDY, S.T.; WANG, C.Y.; SAKHAEE, K.; BRINKLEY, L. \& PAK, C.Y. Effect of lowcarbohydrate high-protein diets on acid-base balance, stone-forming propensity, and calcium metabolism. Am J Kidney Dis. 40: 265 -74, 2002.

REGO, R.A., BERARDO, F.A., RODRIGUES, S.S. e cols.. Risk factors for chronic noncommunicable diseases: a domiciliary survey in the municipality of Sao Paulo, SP (Brazil). Methodology and preliminary results. Rev Saúde Pública, 24(4): 277-85, 1990.

SACKS, F.M.; SVETKEY, L.P., VOLLMER, W.M. e cols.. DASH - Sodium Collaborative Research Group. Effects on blood pressure of reduced dietary sodium and the dietary approaches to stop hypertension (DASH) diet. N Engl J Med, 344: 3-10, 2001.

SEMPOS, C.T.; BRIEFEL, R.R.; JOHNSON, C. \& WOTEKI, C.E. Process and rationale for selecting dietary methods for NHANES III. Vital Health Stat 4, 27:85-90, 1992.

SERRA MAJEM, L.; MORALES, D.; DOMINGO, C.; CAUBET, E.; RIBAS, L. \& NOGUES, R.M. Comparison of 2 methods of evaluation of food and nutrient intake: 24-hour recall and semiquantitative frequency questionnaire. Med Clin (Barc) 103: 652-6, 1994.

SOCIEDADE BRASILEIRA DE CARDIOLOGIA. Consenso Brasileiro sobre Dislipidemias. Arq. Bras. Cardiol. 67, 2, 1996.

SOCIEDADE BRASILEIRA DECARDIOLOGIA. Exercício anti-sedentarismo/obesidade [online]. 1999a. [acessado em 14/4/99]. Disponível no endereço: <http:// www.cardiol.br/exerc.htm>.

SOCIEDADE BRASILEIRA DE CARDIOLOGIA. Programa nacional de prevenção e epidemiologia [online] 1999b. [acessado em 14/4/99]. Disponível no endereço: <http:/ /www.cardiol.br/epidemio.htm>.

SOCIEDADE BRASILEIRA DE CARDIOLOGIA. Prevenção da Aterosclerosedislipidemia. Projeto Diretrizes. Agosto, 2001.

SOCIEDADE BRASILEIRA DE HIPERTENSÃO ARTERIAL. III Concenso Brasileiro de Hipertensão Arterial. Campos do Jordão, SP, 12 a 15 de fevereiro de 1998. Disponível em: http:// www.cardio.br/ dha/concenso. [2002 dez 13].

TARASUK, V. \& BEATON, G.H. Statistical estimation of dietary parameters: implications of patterns in within-subject variation - a case study of sampling strategies. Am J Clin Nutr, 55:22-7, 1992.

TAVELLI, S.; BEERMAN, K.A.S. \& JILL, E. Sources of error and nutritional adequacy of food guide pyramid. J Am Coll Health, 47: 77-82, 1998. 
THE TRIALS OF HYPERTENSION PREVENTION COLLABORATIVE RESEARCH GROUP. The effects of nonpharmacologic interventions on blood pressure of persons with high normal levels. Results of the Trials of Hypertension Prevention. Phase I. JAMA, 267, 9, p. 1213-20, 1992.

TOPOROVSKY J, TOPOROVSKY MS. Arterial hypertension in childhood. Rev Paul Med., 100(1):20-3, 1982.

USDA-UNITED STATES DEPARTMENT OFAGRICULTURE. The food guide pyramid. Washington, DC.1992.

USA, 1989 - Department of Health and Human Services. Nutrition monitoring in the United States- An update report on nutrition monitoring. Washington, DC: Government Printing Office.

VOLLMER, W.M.; SACKS, F.M. \& SVETKEY, L.P. New insights into the effects on blood pressure of diets low in salt and high in fruits and vegetables and low-fat dairy products. Curr Control Trials Cardiovasc. Med., 2:71-74, 2001.

WESTERTERP-PLANTEGA, M.S., WIJCKMANS-DUIJSENS, N.E.G., VERBOEKETVan de VENNE, W.P.G., GRAAF, K.H.,WESTSTRATE, J.A. Energy intake and body weight effects of six months reduced or full fat diets, as a function of dietary restraint. International Journal of Obesity, 22, 1,p.14-22, 1998.

WILLETT W.C. Diet, nutrition, and avoidable cancer. Environmental Health Perspectives; 103, 8:165-70, 1995.

WILLETT, W. Nutritional Epidemiology. $2^{\text {nd }}$ ed. New York, NY: Oxford University Press; 1998.

WHO,. THE WORLD HEALTH REPORT 1997 - executive summary - Conquering Suffering Enriching Humanity World Health Organization - Geneva, 1997.

WHO. Obesity: preventing and managing the global endemic. Report of a WHO consultation. Geneva: World Health Organization. [WHO Technical Report Series, 894], , 2000.

WHO. Diet, physical activity and health. Geneva, World Health Organization,2002

WHO. Diet, nutrition and the prevention of chronic diseases. Report of a Joint WHO/FAO Expert Consultation. Geneva: World Health Organization. [WHO Technical Report Series, 916], 2003.

WING, R.R.; GOLDSTEIN, M.G.; ACTON, K.J.; BIRCH, L.L.; JAKICIC, J.M.; SALLIS, J.F.; SMITH-WEST, D.; JEFFERY, R.W. \& SURWIT, R.S. Behavioral Science Research in Diabetes: Lifestyle changes related to obesity, eating behavior and physical activity. Diabetes Care; 24:117-123, 2001. 
Envie suas publicações para o Conselho Editorial da revista Universitas - Ciências da Saúde.

\author{
Endereço para envio: \\ SEPN 707/907, Campus do UniCEUB \\ 70790-075 Brasília-DF
}

universitas.saude@uniceub.br

Verifique as normas de publicação no final deste número! 